US Army Corps

of Engineers ${ }_{\circledast}$

Engineer Research and

Development Center

Engineered Resilient Systems

\title{
Integrated Rule-Oriented Data System (iRODS) and High Performance Computing (HPC) Architecture Design
}

Kevin D. Winters, Mark A. Cowan, Glover E. George,

Megan E. Gonzalez, Brian Priest, Omar Morris,

and Jonathan Landrum

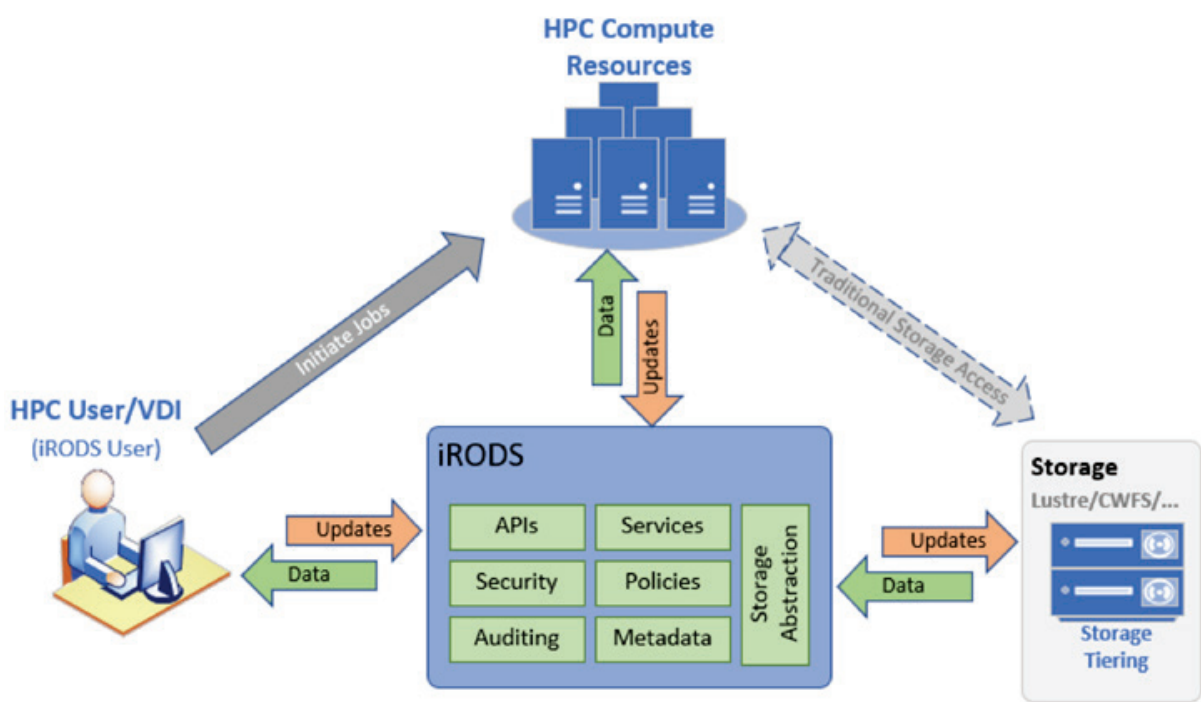


The U.S. Army Engineer Research and Development Center (ERDC) solves the nation's toughest engineering and environmental challenges. ERDC develops innovative solutions in civil and military engineering, geospatial sciences, water resources, and environmental sciences for the Army, the Department of Defense, civilian agencies, and our nation's public good. Find out more at www.erdc.usace.army.mil.

To search for other technical reports published by ERDC, visit the ERDC online library at http://acwc.sdp.sirsi.net/client/default. 


\section{Integrated Rule-Oriented Data System (iRODS) and High Performance Computing (HPC) Architecture Design}

Kevin D. Winters, Mark A. Cowan, Glover E. George, and Megan E. Gonzalez

U.S. Army Engineer Research and Development Center (ERDC)

Information Technology Laboratory (ITL)

3909 Halls Ferry Road

Vicksburg, MS 39180-6199

Brian Priest, Omar Morris, and Jonathan Landrum

Geocent Jackson Mississippi Office

1000 Highland Colony Parkway, Suite 5203

Ridgeland, MS 39157

Final Report

Approved for public release; distribution is unlimited.

Prepared for Headquarters, U.S. Army Corps of Engineers

Washington, DC 20314-1000

Under Work Unit 92L5D8 


\section{Abstract}

The Integrated Rule-Oriented Data System (iRODS) proof-of-concept will be deployed within the existing U.S. Army Engineer Research and Development Center (ERDC) Department of Defense Supercomputing Resource Center (DSRC) to test additional capabilities and features for high performance computing (HPC) users. iRODS is a data-grid middleware that virtualizes access to data, regardless of which physical storage device the data resides within. Users, and HPC jobs on behalf of users, can leverage the various application programming interfaces (APIs) within iRODS to search and retrieve data using metadata and a unified data namespace. In addition to facilitating data discovery and retrieval, iRODS has a robust security system to implement fine-grained access control and auditing rules. 


\section{Contents}

Abstract...................................................................................................................................... if

Figures and Tables......................................................................................................................iv

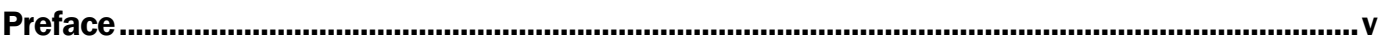

1 Introduction.................................................................................................................................... 1

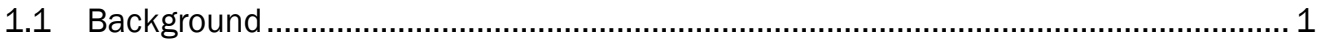

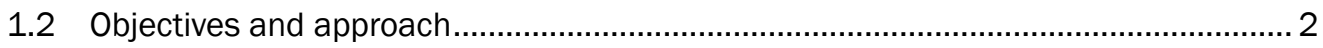

1.3 Project scope................................................................................................ 2

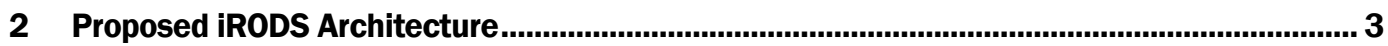

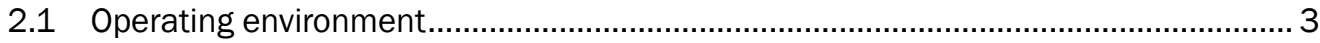

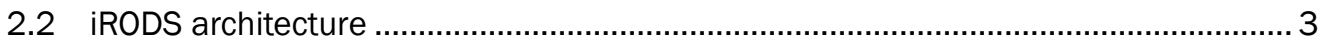

2.3 Hardware .......................................................................................................... 4

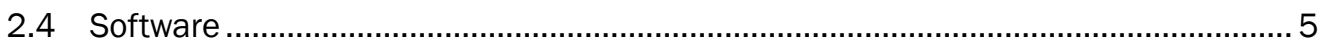

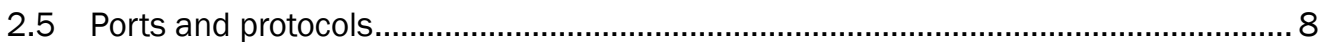

2.6 Security technical implementation guides (STIGS) ............................................... 10

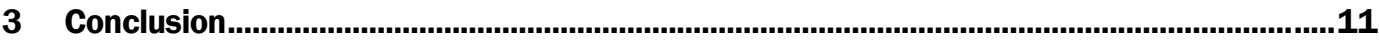

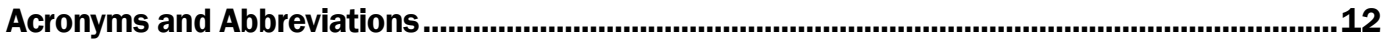

Report Documentation Page 


\section{Figures and Tables}

\section{Figures}

Figure 1-1. iRODS proof-of-concept conceptual diagram................................................................. 1

Figure 2-1. iRODS prototype architecture diagram. .......................................................................... 4

\section{Tables}

Table 2-1. iRODS prototype hosts. . 4

Table 2-2. System software per host. 5

Table 2-3. Ports and protocols per host. 8 


\section{Preface}

This is a deliverable product under the Engineered Resilient Systems (ERS) Program, Data Analytics Work Package, Collaborative Tradespace Analytics Work Unit 92L5D8. Dr. Owen J. Eslinger was the Program Manager, and Dr. Robert M. Wallace was the Technical Director of the ERS program.

The work was performed by the Computational Analysis Branch (CAB) of the Computational Science and Engineering Division (CSED), U.S. Army Engineer Research and Development Center (ERDC), Information Technology Laboratory (ITL), Vicksburg, MS. At the time of publication, Dr. Jeffrey L. Hensley was Chief, CAB; Dr. Jerrell R. Ballard, Jr. was the Chief, CSED; Ms. Patti S. Duett was the Deputy Director of ITL and the Director was Dr. David A. Horner.

COL Teresa A. Schlosser was the Commander of ERDC, and Dr. David W. Pittman was the Director. 


\section{Introduction}

\subsection{Background}

The Integrated Rule-Oriented Data System (iRODS) proof-of-concept will be deployed within the existing U.S. Army Engineer Research and Development Center (ERDC) Department of Defense Supercomputing Resource Center (DSRC) to test additional capabilities and features for high performance computing (HPC) users. iRODS (as seen in Figure 1-1) is a data-grid middleware that virtualizes access to data, regardless of which physical storage device the data resides within. Users, and HPC jobs on behalf of users, can leverage the various application programming interfaces (APIs) within iRODS to search and retrieve data using metadata and a unified data namespace. In addition to facilitating data discovery and retrieval, iRODS has a robust security system to implement finegrained access control and auditing rules.

Figure 1-1. iRODS proof-of-concept conceptual diagram.

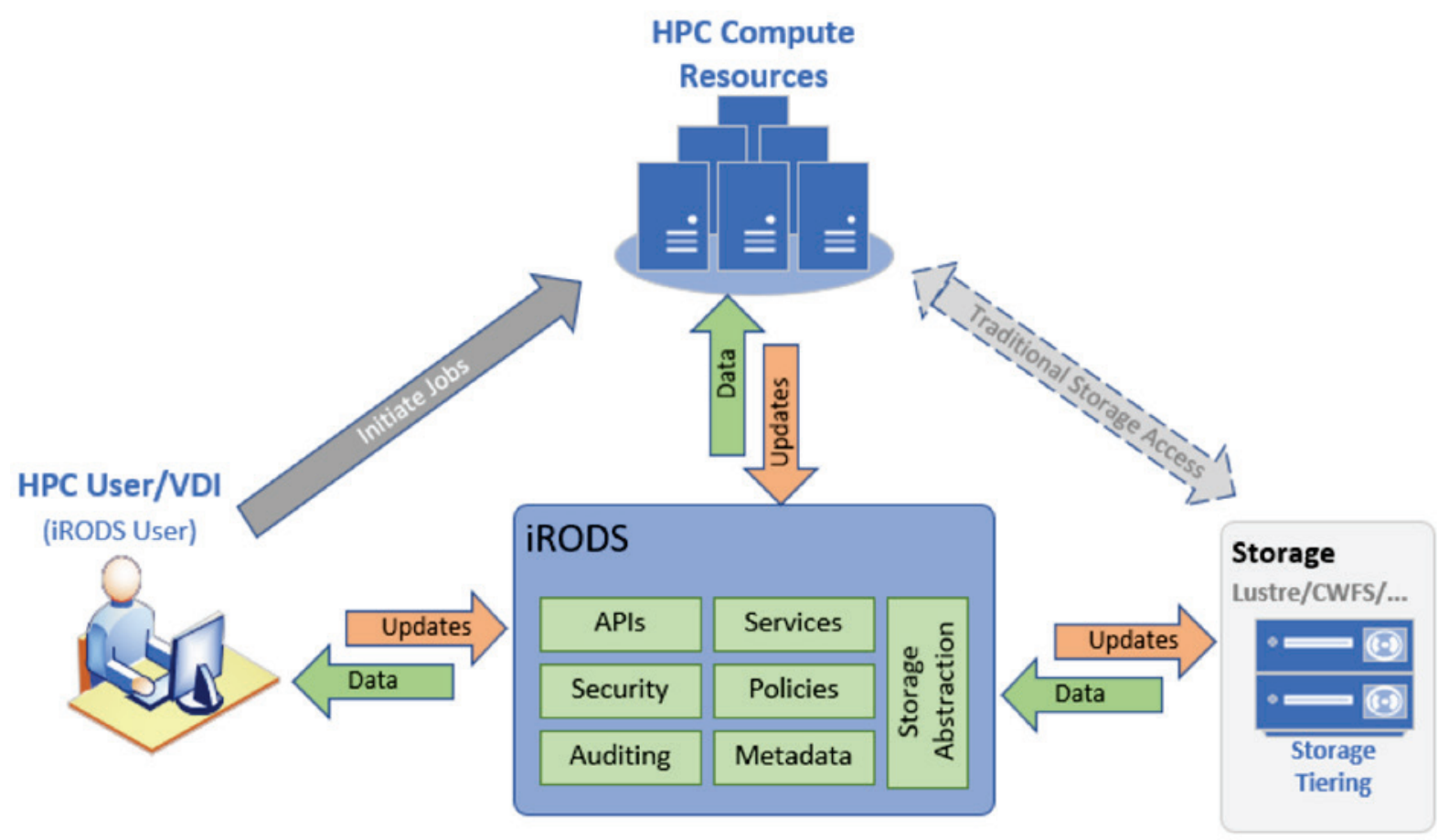




\subsection{Objectives and approach}

This report will outline the architectural design for the implementation of iRODS within the HPC system. To do this, the technical details of the operating environment, the iRODS architecture, the associated hardware, software, and the servers required will be outlined.

\subsection{Project scope}

The architecture design presented within this report is the result of the research phase conducted for the ERDC iRODS project with requirements input from multiple Engineered Resilient Systems (ERS) teams. Specifically, the Data Analytics Team, the Environmental Simulation Team, the Sensor Systems Research Team, and the HPC/Scientific computing group, representing the "general HPC user," all contributed use cases and requirements that lead to the proposed architecture design for the iRODS proof-of-concept phase. 


\section{Proposed iRODS Architecture}

\subsection{Operating environment}

Initially, the iRODS prototype will be built and configured within a test network to ensure all software is configured correctly, applicable security technical implementation guides (STIGs) are applied, and functional testing of available components is complete. During this testing phase, iRODS- 5 and iRODS-6, which leverage storage from ONYX Lustre and the center-wide file system (CWFS), respectively, will not be tested. Once the security assessment is complete, the iRODS prototype will be moved to production and testing of iRODS- 5 and iRODS-6. Figure 2-1 shows the production architecture diagram of the iRODS prototype.

\section{2 iRODS architecture}

The individual host components (i.e., virtual machines) for iRODS and the required ports and protocols within and across the iRODS test subnet (i.e., iRODS boundary) are shown in Figure 2-1.

Network access control lists (ACLs) will be implemented so communication across the iRODS test subnet is strictly controlled and within the bounds of the testing required for the iRODS proof-of-concept test event. Users, and HPC resources running jobs (e.g., scripts to retrieve data), will be able to query and retrieve data from iRODS, however, iRODS components will not be able to initiate communication outside of the iRODS test subnet. 
Figure 2-1. iRODS prototype architecture diagram.

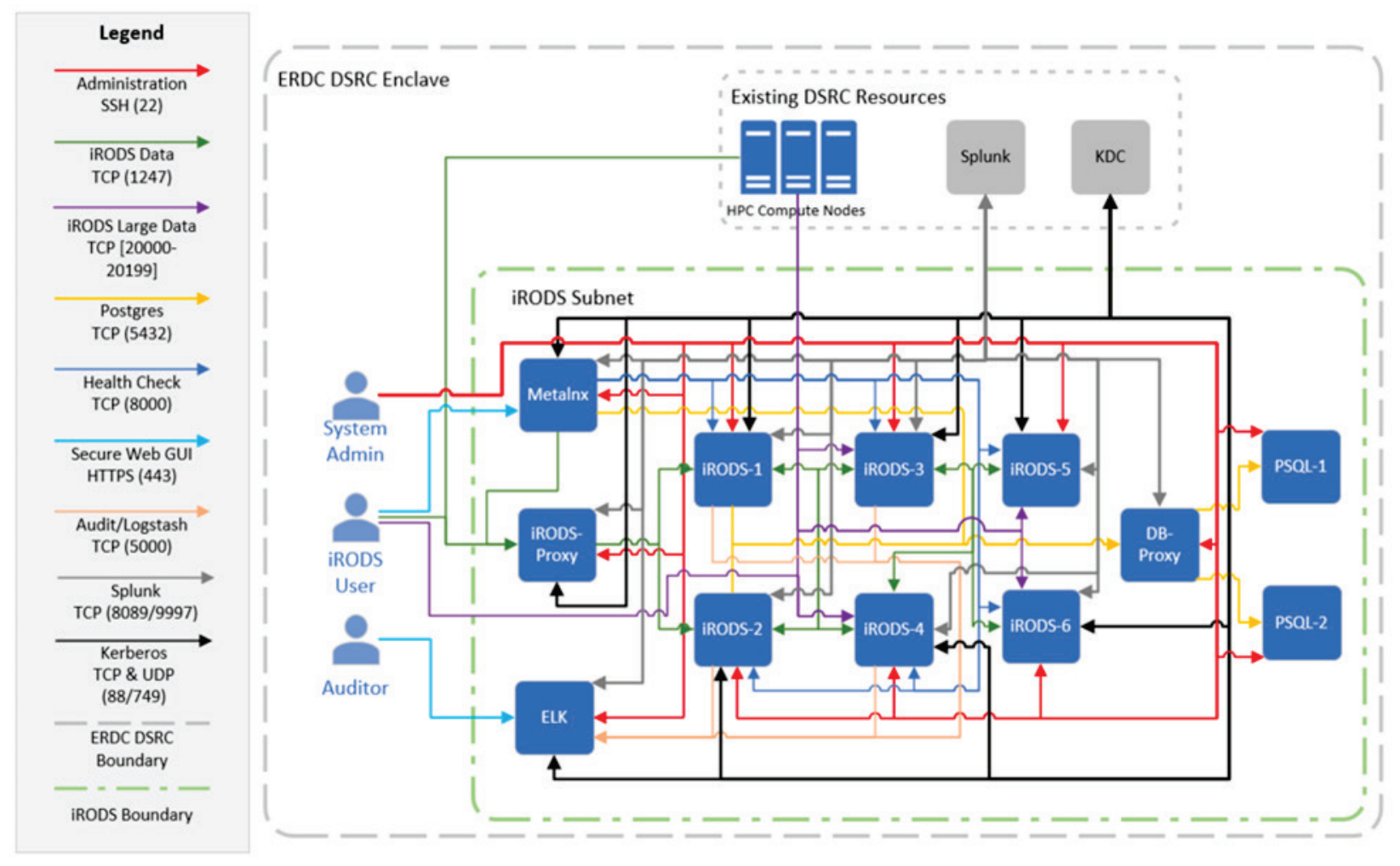

\subsection{Hardware}

The identified hosts and specifications for the iRODS virtual machine prototype are listed in Table 2-1.

Table 2-1. iRODS prototype hosts.

\begin{tabular}{|l|l|l|l|l|}
\hline Server Name & Server Role & CPUs & RAM & Disk Storage \\
\hline iRODS-Proxy & Load Balancer & 16 & $16 \mathrm{~GB}$ & $50 \mathrm{~GB}$ \\
\hline iRODS-1 & iCAT Server & 8 & $8 \mathrm{~GB}$ & $50 \mathrm{~GB}$ \\
\hline iRODS-2 & iCAT Server & 8 & $8 \mathrm{~GB}$ & $50 \mathrm{~GB}$ \\
\hline iRODS-3 & Resource Server & 16 & $16 \mathrm{~GB}$ & $50 \mathrm{~TB}$ \\
\hline iRODS-4 & Resource Server & 16 & $16 \mathrm{~GB}$ & $50 \mathrm{~TB}$ \\
\hline iRODS-5 & Resource Server & 16 & $16 \mathrm{~GB}$ & $500 \mathrm{~TB}$ \\
\hline iRODS-6 & Resource Server & 16 & $16 \mathrm{~GB}$ & $500 \mathrm{~TB} *$ \\
\hline ELK & Elastic Stack & 16 & $32 \mathrm{~GB}$ & $500 \mathrm{~GB}$ \\
\hline Metalnx & iRODS GUI & 8 & $8 \mathrm{~GB}$ & $50 \mathrm{~GB}$ \\
\hline DB-Proxy & DB Load Balancer & 32 & $16 \mathrm{~GB}$ & $50 \mathrm{~GB}$ \\
\hline PSQL-1 & Database Server & 32 & $128 \mathrm{~GB}$ & $500 \mathrm{~GB}$ \\
\hline PSQL-2 & Database Server & 32 & $128 \mathrm{~GB}$ & $500 \mathrm{~GB}$ \\
\hline
\end{tabular}

* iRODS-5 storage must be provided by ONYX Lustre filesystem (i.e., iRODS vault on ONYX).

** iRODS- 6 storage must be provided by CWFS (i.e., iRODS vault on CWFS). 


\subsection{Software}

The software and version for each identified host for the iRODS prototype is listed in Table 2-2.

Table 2-2. System software per host.

\begin{tabular}{|c|c|c|}
\hline Server Name & Software & Version \\
\hline \multirow{4}{*}{ PSQL-1 } & RHEL & 7 \\
\hline & PostgreSQL & 10.3 \\
\hline & Splunk Universal Forwarder & 7.2 .3 \\
\hline & HPCMP Kerberos Kit & Latest Version \\
\hline \multirow{4}{*}{ PSQL-2 } & RHEL & 7 \\
\hline & PostgreSQL & 10.3 \\
\hline & Splunk Universal Forwarder & 7.2 .3 \\
\hline & HPCMP Kerberos Kit & Latest Version \\
\hline \multirow{12}{*}{ ¡RODS-1 } & RHEL & 7 \\
\hline & iRODS & 4.2 .4 \\
\hline & irods-rule-engine-plugin-audit-amqp & 4.2 .4 \\
\hline & irods-externals-qpid-with-proton 0.34 & 4.2 .4 \\
\hline & Python & 3.7 .2 \\
\hline & python-irodsclient & 4.2 .4 \\
\hline & irods-rule-engine-plugin-python & 4.2 .4 \\
\hline & irods-database-plugin-postgres & 4.2 .4 \\
\hline & irods-rule-engine-plugin-storage-tiering & 4.2 .4 \\
\hline & Metalnx Remote Monitor Daemon & $2.0-0$ \\
\hline & Splunk Universal Forwarder & 7.2 .3 \\
\hline & HPCMP Kerberos Kit & Latest Version \\
\hline \multirow{12}{*}{ iRODS-2 } & RHEL & 7 \\
\hline & iRODS & 4.2 .4 \\
\hline & irods-rule-engine-plugin-audit-amqp & 4.24 \\
\hline & irods-externals-qpid-with-proton 0.34 & 4.2 .4 \\
\hline & Python & 3.7 .2 \\
\hline & python-irodsclient & 4.2 .4 \\
\hline & irods-rule-engine-plugin-python & 4.2 .4 \\
\hline & irods-database-plugin-postgres & 4.2 .4 \\
\hline & irods-rule-engine-plugin-storage-tiering & 4.2 .4 \\
\hline & Metalnx Remote Monitor Daemon & $2.0-0$ \\
\hline & Splunk Universal Forwarder & 7.2 .3 \\
\hline & HPCMP Kerberos Kit & Latest Version \\
\hline
\end{tabular}




\begin{tabular}{|c|c|c|}
\hline Server Name & Software & Version \\
\hline \multirow{14}{*}{ iRODS-3 } & RHEL & 7 \\
\hline & iRODS & 4.2 .4 \\
\hline & irods-rule-engine-plugin-audit-amqp & 4.2 .4 \\
\hline & Python & 3.7 .2 \\
\hline & python-irodsclient & 4.2 .4 \\
\hline & irods-rule-engine-plugin-python & 4.2 .4 \\
\hline & irods-database-plugin-postgres & 4.2 .4 \\
\hline & irods-rule-engine-plugin-storage-tiering & 4.2 .4 \\
\hline & Metalnx Remote Monitor Daemon & $2.0-0$ \\
\hline & Redis & 5.0 .3 \\
\hline & Pip & 8.1 .1 \\
\hline & irods-capability-automated-ingest & 0.3 .4 \\
\hline & Splunk Universal Forwarder & 7.2 .3 \\
\hline & HPCMP Kerberos Kit & Latest Version \\
\hline \multirow{14}{*}{ ¡RODS-4 } & RHEL & 7 \\
\hline & iRODS & 4.2 .4 \\
\hline & irods-rule-engine-plugin-audit-amqp & 4.2 .4 \\
\hline & Python & 3.7 .2 \\
\hline & python-irodsclient & 4.2 .4 \\
\hline & irods-rule-engine-plugin-python & 4.2 .4 \\
\hline & irods-database-plugin-postgres & 4.2 .4 \\
\hline & irods-rule-engine-plugin-storage-tiering & 4.2 .4 \\
\hline & Metalnx Remote Monitor Daemon & $2.0-0$ \\
\hline & Redis & 5.0 .3 \\
\hline & Pip & 8.1 .1 \\
\hline & irods-capability-automated-ingest & 0.3 .4 \\
\hline & Splunk Universal Forwarder & 7.2 .3 \\
\hline & HPCMP Kerberos Kit & Latest Version \\
\hline \multirow{12}{*}{ ¡RODS-5 } & RHEL & 7 \\
\hline & iRODS & 4.2 .4 \\
\hline & irods-rule-engine-plugin-audit-amqp & 4.2 .4 \\
\hline & Python & 3.7 .2 \\
\hline & python-irodsclient & 4.2 .4 \\
\hline & irods-rule-engine-plugin-python & 4.2 .4 \\
\hline & irods-database-plugin-postgres & 4.2 .4 \\
\hline & irods-rule-engine-plugin-storage-tiering & 4.2 .4 \\
\hline & Metalnx Remote Monitor Daemon & $2.0-0$ \\
\hline & Redis & 5.0 .3 \\
\hline & Pip & 8.1 .1 \\
\hline & irods-capability-automated-ingest & 0.3 .4 \\
\hline
\end{tabular}




\begin{tabular}{|c|c|c|}
\hline Server Name & Software & Version \\
\hline & Splunk Universal Forwarder & 7.2 .3 \\
\hline & HPCMP Kerberos Kit & Latest Version \\
\hline \multirow{14}{*}{ iRODS-6 } & RHEL & 7 \\
\hline & iRODS & 4.2 .4 \\
\hline & irods-rule-engine-plugin-audit-amqp & 4.2 .4 \\
\hline & Python & 3.7 .2 \\
\hline & python-irodsclient & 4.2 .4 \\
\hline & irods-rule-engine-plugin-python & 4.2 .4 \\
\hline & irods-database-plugin-postgres & 4.2 .4 \\
\hline & irods-rule-engine-plugin-storage-tiering & 4.2 .4 \\
\hline & Metalnx Remote Monitor Daemon & $2.0-0$ \\
\hline & Redis & 5.0 .3 \\
\hline & Pip & 8.1 .1 \\
\hline & irods-capability-automated-ingest & 0.3 .4 \\
\hline & Splunk Universal Forwarder & 7.2 .3 \\
\hline & HPCMP Kerberos Kit & Latest Version \\
\hline \multirow[t]{4}{*}{ iRODS-Proxy } & RHEL & 7 \\
\hline & HAProxy & 1.7 \\
\hline & Splunk Universal Forwarder & 7.2 .3 \\
\hline & HPCMP Kerberos Kit & Latest Version \\
\hline \multirow{6}{*}{ Metalnx } & RHEL & 7 \\
\hline & Metalnx & 2.0 .0 \\
\hline & Java & 1.8 \\
\hline & Tomcat & 9.0 .14 \\
\hline & Splunk Universal Forwarder & 7.2 .3 \\
\hline & HPCMP Kerberos Kit & Latest Version \\
\hline \multirow{4}{*}{ DB-Proxy } & RHEL & 7 \\
\hline & HAProxy & 1.7 \\
\hline & Splunk Universal Forwarder & 7.2 .3 \\
\hline & HPCMP Kerberos Kit & Latest Version \\
\hline \multirow{9}{*}{ ELK } & RHEL & 7 \\
\hline & Java & 1.8 \\
\hline & Active MQ & 5.15 .8 \\
\hline & Elastic Search & 6.5 .4 \\
\hline & Logstash & 6.5 .4 \\
\hline & Kibana & 6.5 .4 \\
\hline & Stomp-plugin & 3.0 .8 \\
\hline & Splunk Universal Forwarder & 7.2 .3 \\
\hline & HPCMP Kerberos Kit & Latest Version \\
\hline
\end{tabular}




\subsection{Ports and protocols}

The required ports and protocols utilized by the iRODS proof-of-concept architecture are listed in Table 2-3.

Table 2-3. Ports and protocols per host.

\begin{tabular}{|c|c|c|c|c|}
\hline Source & Destination & Protocol & Port & Notes \\
\hline$<$ Sys Admin > & $\begin{array}{l}\text { ELK } \\
\text { iRODS-Proxy } \\
\text { iRODS-1 } \\
\text { iRODS-2 } \\
\text { iRODS-3 } \\
\text { iRODS-4 } \\
\text { iRODS-5 } \\
\text { iRODS-6 } \\
\text { Metalnx } \\
\text { DB-Proxy } \\
\text { PSQL-1 } \\
\text { PSQL-2 }\end{array}$ & $\mathrm{SSH}$ & 22 & $\begin{array}{l}\text { Remote system administration via } \\
\text { command line }\end{array}$ \\
\hline $\begin{array}{l}\text { iRODS-1 } \\
\text { iRODS-2 } \\
\text { iRODS-3 } \\
\text { iRODS-4 } \\
\text { iRODS-5 } \\
\text { iRODS-6 }\end{array}$ & $\begin{array}{l}\text { iRODS-1 } \\
\text { iRODS-2 } \\
\text { iRODS-3 } \\
\text { iRODS-4 } \\
\text { iRODS-5 } \\
\text { iRODS-6 }\end{array}$ & TCP & 1247 & Inter-sever communication \\
\hline$<$ iRODS User> & iRODS-Proxy & TCP & 1247 & Load balancing (iCommands, Metalnx GUI) \\
\hline iRODS-Proxy & $\begin{array}{l}\text { iRODS-1 } \\
\text { iRODS-2 }\end{array}$ & TCP & 1247 & Load balancing (iCommands, Metalnx GUI) \\
\hline$<$ iRODS Admin $>$ & $\begin{array}{l}\text { iRODS-1 } \\
\text { iRODS-2 } \\
\text { iRODS-3 } \\
\text { iRODS-4 } \\
\text { iRODS-5 } \\
\text { iRODS-6 }\end{array}$ & TCP & 1248 & $\begin{array}{l}\text { iRODS Data grid tasks (ex: status, pause, } \\
\text { resume, shutdown) }\end{array}$ \\
\hline $\begin{array}{l}\text { iRODS-1 } \\
\text { iRODS-2 }\end{array}$ & DB-Proxy & TCP & 5432 & iCAT Database communication \\
\hline DB-Proxy & $\begin{array}{l}\text { PSQL-1 } \\
\text { PSQL-2 }\end{array}$ & TCP & 5432 & Database communication \\
\hline$<$ iRODS User $>$ & $\begin{array}{l}\text { iRODS-3 } \\
\text { iRODS-4 } \\
\text { iRODS-5 } \\
\text { iRODS-6 }\end{array}$ & TCP & $\begin{array}{l}20000 \\
-20199\end{array}$ & $\begin{array}{l}\text { Port range for files }>32 \mathrm{MB} \text { multiple thread } \\
\text { parallel transfer }\end{array}$ \\
\hline Metalnx & iRODS-Proxy & TCP & 1247 & iCAT Load balancing \\
\hline Metalnx & DB-Proxy & TCP & 5432 & Metalnx Database communication \\
\hline
\end{tabular}




\begin{tabular}{|c|c|c|c|c|}
\hline Source & Destination & Protocol & Port & Notes \\
\hline Metalnx & $\begin{array}{l}\text { iRODS-1 } \\
\text { iRODS-2 } \\
\text { iRODS-3 } \\
\text { iRODS-4 } \\
\text { iRODS-5 } \\
\text { iRODS-6 }\end{array}$ & TCP & 8000 & $\begin{array}{l}\text { Remote Monitoring Daemon } \\
\text { communication }\end{array}$ \\
\hline <iRODS User> & Metalnx & HTTPS & 443 & iRODS Web interface \\
\hline <Auditor> & ELK & HTTPS & 443 & Kibana Web Interface \\
\hline $\begin{array}{l}\text { iRODS-1 } \\
\text { iRODS-2 } \\
\text { iRODS-3 } \\
\text { iRODS-4 } \\
\text { iRODS-5 } \\
\text { iRODS-6 }\end{array}$ & ELK & TCP & 5000 & Logstash \\
\hline $\begin{array}{l}\text { ELK } \\
\text { HA-Proxy } \\
\text { iRODS-1 } \\
\text { iRODS-2 } \\
\text { iRODS-3 } \\
\text { iRODS-4 } \\
\text { iRODS-5 } \\
\text { iRODS-6 } \\
\text { Metalnx } \\
\text { DB-Proxy } \\
\text { PSQL-1 } \\
\text { PSQL-2 }\end{array}$ & Splunk Server & TCP & $\begin{array}{l}8089 \\
9997\end{array}$ & $\begin{array}{l}\text { Splunkd } \\
\text { Splunk indexer }\end{array}$ \\
\hline $\begin{array}{l}\text { ELK } \\
\text { HA-Proxy } \\
\text { iRODS-1 } \\
\text { iRODS-2 } \\
\text { iRODS-3 } \\
\text { iRODS-4 } \\
\text { iRODS-5 } \\
\text { iRODS-6 } \\
\text { Metalnx } \\
\text { PSQL }\end{array}$ & Kerberos Server & $\begin{array}{l}\text { TCP/UPD } \\
\text { TCP/UDP }\end{array}$ & $\begin{array}{l}88 \\
749\end{array}$ & $\begin{array}{l}\text { Kerberos KDC } \\
\text { Kerberos KDC }\end{array}$ \\
\hline
\end{tabular}




\subsection{Security technical implementation guides (STIGS)}

The following STIG or Security Requirements Guides (SRG) will be implemented for each host server containing the associated software components:

- Application Security and Development STIG - Ver 4, Rel 9

- Application Server SRG - Ver 2, Rel 5

- Oracle JRE 8 UNIX STIG - Ver 1, Rel 3

- PostgreSQL 9.x STIG- Ver 1, Rel 4

- Red Hat 6 STIG - Ver 1, Rel 21

- Web Server Security Requirements Guide (SRG) - Ver 2, Rel 2. 


\section{Conclusion}

This report presents the integrated iRODS and HPC architecture design. This includes the technical details of the operating environment, the iRODS architecture, the associated hardware, software, and the minimum servers required. 


\section{Acronyms and Abbreviations}

\begin{tabular}{|l|l|}
\hline Acronym & \multicolumn{1}{|c|}{ Meaning } \\
\hline ACL & Access Control Lists \\
\hline APIs & Application Programming Interfaces \\
\hline CWFS & Center-Wide File System \\
\hline DoD & Department of Defense \\
\hline DSRC & Defense Supercomputing Resource Center \\
\hline ERDC & Engineer Research and Development Center \\
\hline ERS & Engineered Resilient Systems \\
\hline HPC & High-Performance Computing \\
\hline IA & Information Assurance \\
\hline iRODS & Integrated Rule-Oriented Data System \\
\hline SRG & Security Requirements Guides \\
\hline STIGS & Security Technical Implementation Guide \\
\hline USACE & U.S. Army Corps of Engineers \\
\hline
\end{tabular}




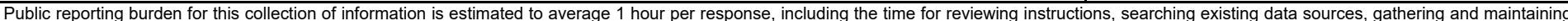

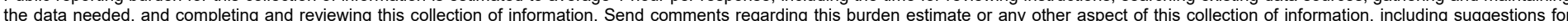

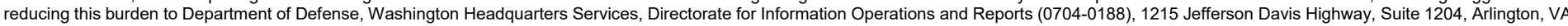

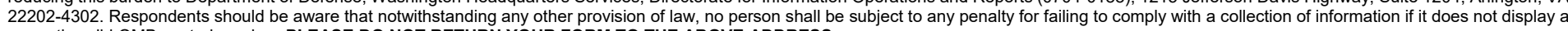
currently valid OMB control number. PLEASE DO NOT RETURN YOUR FORM TO THE ABOVE ADDRESS.
1. REPORT DATE $(D D-M M-Y Y Y Y)$
September 2020
2. REPORT TYPE
Final report

\section{TITLE AND SUBTITLE}

Integrated Rule-Oriented Data System (iRODS) and High Performance Computing (HPC)

Architecture Design

\section{DATES COVERED (From - To)}

5a. CONTRACT NUMBER

5b. GRANT NUMBER

5c. PROGRAM ELEMENT NUMBER

\section{AUTHOR(S)}

Kevin D. Winters, Mark A. Cowan, Glover E. George, Megan E. Gonzalez, Brian Priest, Omar Morris, and Jonathan Landrum

\section{5d. PROJECT NUMBER}

5e. TASK NUMBER

5f. WORK UNIT NUMBER

92L5D8

\section{PERFORMING ORGANIZATION NAME(S) AND ADDRESS(ES)}

8. PERFORMING ORGANIZATION REPORT NUMBER

U.S. Army Engineer Research and Development Center

Information Technology Laboratory

ERDC/ITL SR-20-12

3909 Halls Ferry Road, Vicksburg, MS 39180-6199

\section{SPONSORING / MONITORING AGENCY NAME(S) AND ADDRESS(ES)}

10. SPONSOR/MONITOR'S ACRONYM(S)

Headquarters, U.S. Army Corps of Engineers

Washington, DC 20314-1000

\section{SPONSOR/MONITOR'S REPORT}

NUMBER(S)

\section{DISTRIBUTION / AVAILABILITY STATEMENT}

Approved for public release; distribution unlimited.

\section{SUPPLEMENTARY NOTES}

\section{ABSTRACT}

The Integrated Rule-Oriented Data System (iRODS) proof-of-concept will be deployed within the existing U.S. Army Engineer Research and Development Center (ERDC) Department of Defense Supercomputing Resource Center (DSRC) to test additional capabilities and features for high performance computing (HPC) users. iRODS is a data-grid middleware that virtualizes access to data, regardless of which physical storage device the data resides within. Users, and HPC jobs on behalf of users, can leverage the various application programming interfaces (APIs) within iRODS to search and retrieve data using metadata and a unified data namespace. In addition to facilitating data discovery and retrieval, iRODS has a robust security system to implement fine-grained access control and auditing rules.

15. SUBJECT TERMS

iRODS (Computer system)

High performance computing

Computer architecture

Computer science

\section{SECURITY CLASSIFICATION OF:}

a. REPORT

UNCLASSIFIED

b. ABSTRACT
UNCLASSIFIED

c. THIS PAGE

UNCLASSIFIED

\begin{tabular}{|c|c|} 
17. LIMITATION & $\begin{array}{c}\text { 18. NUMBER } \\
\text { OF ABSTRACT }\end{array}$ \\
OF PAGES \\
\end{tabular}

19a. NAME OF RESPONSIBLE PERSON

19b. TELEPHONE NUMBER (include area code) 